\title{
Effect of cholesterol supplementation on cryosurvival of goat spermatozoa
}

\author{
Sunita Behera ${ }^{1}$, Hiron M. Harshan², K. Lekshmi Bhai and K. N. Aravinda Ghosh ${ }^{3}$
}

1. Department of Veterinary Gynaecology and Obstetrics, Veterinary College, Bengaluru, Karnataka, India; 2. Department of Animal Reproduction Gynaecology and Obstetrics, College of Veterinary and Animal Sciences, Kerala Veterinary and Animal Sciences University, Pookode, Kerala, India; 3. Department of Animal Reproduction, Gynaecology and Obstetrics, College of Veterinary and Animal Sciences, Kerala Veterinary and Animal Sciences University, Mannuthy, Kerala, India.

Corresponding author: Sunita Behera, e-mail: sunita5656@gmail.com, HMH: hiron@rediffmail.com, KLB: bhai04vet@gmail.com, KNAG: ghosh@kvasu.ac.in

Received: 07-06-2015, Revised: 24-10-2015, Accepted: 31-10-2015, Published: 12-12-2015

doi: 10.14202/vetworld.2015.1386-1391 How to cite this article: Behera S, Harshan HM, Bhai KL, Ghosh KNA (2015) Effect of cholesterol supplementation on cryosurvival of goat spermatozoa, Veterinary World 8(12): 1386-1391.

\begin{abstract}
Aim: Sperm membrane cholesterol influences cryodamage during cryopreservation. The present study was carried out to evaluate the effect of varying cholesterol levels in Tris based extenders on the freezability of sexually healthy Malabari buck semen.

Materials and Methods: A total of 48 ejaculates from two adults healthy sexually healthy Malabari bucks were utilized for the study. The collected and pooled ejaculates were divided into four groups with Group I serving as Control - I, Group II and III were treated with $1 \mathrm{mg}$ and $2 \mathrm{mg}$ of cholesterol-loaded-cyclodextrin (CLC)/120 × 10 6 spermatozoa, respectively, and Group IV treated with $1 \mathrm{mg}$ methyl- $\beta$-cyclodextrin $(\mathrm{M} \beta \mathrm{CD})$ served as Control - II. Manual freezing was carried out to cryopreserve the treated and control spermatozoa.

Results: Treatment of semen samples with CLC resulted in improved maintenance of sperm motility at pre-freeze and post-thaw stages of cryopreservation without affecting hypo-osmotic swelling response. Treatment of semen with $1 \mathrm{mg}$ of $\mathrm{CLC} / 120 \times 10^{6}$ spermatozoa was observed to be better than treatment with $2 \mathrm{mg}$ of CLC $/ 120 \times 10^{6}$ spermatozoa. In general, $\mathrm{M} \beta C D$ treatment was found to result in significantly lower sperm characteristics than those of Control - I and CLC treatment at pre-feeze and post-thaw stages and when incubated up to $4 \mathrm{~h}$.
\end{abstract}

Conclusion: Cholesterol treatment of sexually healthy Malabari buck semen was found to hold promise for improving cryopreservability of spermatozoa.

Keywords: buck, cholesterol, cryopreservation, functional membrane integrity, motility, spermatozoa.

\section{Introduction}

Artificial insemination (AI) has become an inevitable tool in the breeding management of many of the domestic animals. Though, AI has been successfully implemented among the cattle population; it has not been as successful among the goats. Irrespective of many years of research, fertility of frozen semen remains one of the limitations in its acceptability in goat breeding.

Sperm damage occurring during cryopreservation due to cold shock, low-temperature exposure, osmotic stress, oxidative stress, and combinations of these conditions [1]. It has been suggested that the sperm plasma membrane is the primary site of damage induced by cryopreservation and is one of the main reasons for reduced motility and fertility of sperm during cryopreservation [2,3]. Cholesterol is an important component in the regulation of membrane fluidity, aiding in the stabilization of the membrane [4]. Cold shock induces plasma membrane lipid phase transitions, which can be reduced by increasing the proportion of cholesterol within the plasma membrane [5]. In addition,

Copyright: The authors. This article is an open access article licensed under the terms of the Creative Commons Attributin License (http:// creative commons.org/licenses/by/2.0) which permits unrestricted use, distribution and reproduction in any medium, provided the work is properly cited. cryopreservation induces cholesterol depletion from plasma membrane, which in turn causes membrane destabilization [6]. Buck spermatozoa are a more prone to cold shock because of lower membrane cholesterol to phospholipid ratio compared to rabbit and humans [7]. In recent times, the concept of cholesterol supplementation as a means of increasing sperm membrane cholesterol content and hence freezability has gained acceptance [8]. The increase in the cryosurvival is attributed to the increased membrane fluidity and broadening of phase transition temperature [9]. Cyclodextrins are cyclic oligosaccharides of glucose, if pre-loaded with cholesterol, can insert cholesterol into cell membranes. Several authors have reported increased cryosurvival rates when boars, bull, buck, ram, and stallion spermatozoa were supplemented with cholesterol.

Hence, a study was carried out to evaluate the effect of varying levels of cholesterol in Tris based extenders on the post-thaw motility and plasma membrane intactness of sexually healthy Malabari buck spermatozoa.

\section{Materials and Methods}

\section{Ethical approval}

The study was conducted after approval of research committee and institutional ethical committee. 


\section{Materials}

Cholesterol and methyl- $\beta$-cyclodextrin (M $\beta C D)$ were bioreagent grade and purchased from SigmaAldrich, India. All freezing chemicals were of analytical grade purchased from Merck life sciences, India and stains from Himedia, India.

Preparation of cholesterol-loaded-cyclodextrin (CLC) CLC was prepared according to the method described by Purdy and Graham [10]. In brief, $500 \mathrm{mg}$ of $\mathrm{M} \beta C \mathrm{CD}$ was dissolved in $1.0 \mathrm{ml}$ of methanol in a glass test tube. In another test tube, $200 \mathrm{mg}$ cholesterol was dissolved in $1.0 \mathrm{ml}$ of chloroform. An aliquot of $0.225 \mathrm{ml}$ of cholesterol solution was transferred to the $\mathrm{M} \beta \mathrm{CD}$ solution and stirred to make a clear solution. The prepared solution was then poured into a glass Petri dish. The solvents were removed under a stream of nitrogen gas. The resulting crystals were kept at room temperature for another $24 \mathrm{~h}$ for drying and stored in a glass vial at room temperature until use. The working solution of CLC was prepared by adding $40 \mathrm{mg}$ of CLC to $1.0 \mathrm{ml}$ of tris-citric acid-glucose (TCG) buffer and the solution mixed using a vortex shaker.

\section{Semen collection and cryopreservation}

Semen was collected using artificial vagina from two sexually healthy Malabari bucks (two ejaculates each) maintained at AI center attached to the Department of Animal Reproduction, College of Veterinary and Animal Sciences, Mannuthy, Trissur. Immediately after collection the vials were transferred to a water bath at $37^{\circ} \mathrm{C}$ in the laboratory and all four ejaculates were pooled after preliminary evaluation.

After preliminary evaluation of semen, the sperm concentration of the 48 ejaculates was determined by hemocytometer. Ejaculate having "++++" mass activity having fast distinct swirl formations (on a scale of 0 to +++++ ), more than $80 \%$ of progressive motile sperm, and density "DDDD" were used for the study. The density of semen sample was graded on a scale of D to DDDD, based on visual examination of opacity of a drop of semen taken on a clean, grease free glass slide. Four ejaculates were pooled and were split into four equal treatment aliquots. One of them was control (without CLC treatment, Group - I), other two aliquots were treated with $1 \mathrm{mg}$ and $2 \mathrm{mg}$ CLC/120 million buck spermatozoa (Group - II and Group - III) and last aliquot was treated with $1 \mathrm{mg} \mathrm{M} \beta C D / 120$ million spermatozoa (Group - IV), in TCG buffer and incubated for $15 \mathrm{~min}$. After that each sample was diluted with TCG buffer containing $10 \%(\mathrm{v} / \mathrm{v})$ egg yolk. Glycerolized diluents containing $5 \%(\mathrm{v} / \mathrm{v})$ egg yolk and $12 \%(\mathrm{v} / \mathrm{v})$ glycerol were added in three steps in 10 min interval $1 \mathrm{~h}$ after reaching $5^{\circ} \mathrm{C}$ to obtain 300 million progressive motile sperms $/ \mathrm{ml}$, packed into $0.5 \mathrm{ml}$ French straws and kept for equilibration at $5^{\circ} \mathrm{C}$ for $2 \mathrm{~h}$. The quality of equilibrated pre-freeze semen was analyzed by thawing one straw from each group was thawed in a water bath at $37^{\circ} \mathrm{C}$ for 30 seconds and assessing progressive motility, viability, acrosome integrity, and functional membrane integrity of spermatozoa.

The straws were frozen in liquid nitrogen vapor, $4 \mathrm{~cm}$ above the liquid nitrogen level, for $10 \mathrm{~min}$ and plunged into liquid nitrogen for storage. One straw from each group was thawed, after at least $24 \mathrm{~h}$ of freezing, in water bath at $37^{\circ} \mathrm{C}$ for $30 \mathrm{~s}$ and post-thaw semen quality (progressive motility, viability, acrosome integrity, and functional membrane integrity of spermatozoa in each group).

\section{Evaluation of spermatozoa}

Percentage of progressive motile sperms were estimated by taking a $20 \mu 1$ drop of diluted semen on a clean slide and examined under a microscope $(\times 400)$. A minimum three fields were taken into account for determination of the percentage of progressive motile sperms. Functional membrane intactness of sperm was assessed by hypo-osmotic swelling (HOS) test described by Jeyendran et al. [11]. Two Eppendorff tubes, one containing $0.9 \mathrm{ml}$ of HOS solution (100 $\mathrm{mOsm} / \mathrm{L}$ ), the other $0.9 \mathrm{ml}$ of control solution (300 $\mathrm{mOsm} / \mathrm{L}$ ) were loaded with $0.1 \mathrm{ml}$ of extended semen each and incubated at $37^{\circ} \mathrm{C}$ for $30 \mathrm{~min}$. After incubation, a drop of eosin solution was added to the semen in Eppendorff tubes and a drop of the suspension from the bottom of each tube was taken on a clean, grease free glass slide and a smear were prepared. A minimum of 200 spermatozoa were examined under the high power objective $(\times 400)$ of a microscope. The actual number of spermatozoa with intact plasma membrane was calculated by subtracting the number of positive spermatozoa in the control from HOS positive spermatozoa of the same semen sample.

To know the resistant of the sperm to incubation after thawing the semen sample of each group were incubated at $37^{\circ} \mathrm{C}$ up to $4 \mathrm{~h}$ of thawing and progressive motility and functional membrane integrity of spermatozoa in each group were assessed at $0 \mathrm{~h}, 30 \mathrm{~min}$, $1 \mathrm{~h}, 2 \mathrm{~h}, 3 \mathrm{~h}, 4 \mathrm{~h}$ time interval as described below.

\section{Statistical analysis}

Data were presented as means \pm standard error of mean. Percentage data (progressive motility, HOS positive sperm) was transformed using Arcsine prior to analysis, and the data were analyzed by one-way analysis of variance. Treatment were considered to be different at $\mathrm{p}<0.05$.

\section{Results}

During post-equilibration period spermatozoa treated with $1 \mathrm{mg}$ and $2 \mathrm{mg} \mathrm{CLC} / 120$ million spermatozoa prior to cryopreservation showed significantly higher $(p<0.05)$ percentage progressive motile sperms (Table-1) than spermatozoa of groups without CLC and $\mathrm{M} \beta \mathrm{CD}$ containing group. Group treated with $1 \mathrm{mg}$ of $\mathrm{M} \beta \mathrm{CD} / 120$ million spermatozoa had significantly lower $(\mathrm{p}<0.05)$ percentage progressive motile sperms (Table-1) than other three groups. After cryopreservation, spermatozoa treated with $1 \mathrm{mg}$ CLC/120 million 
spermatozoa before cryopreservation had significantly higher progressive motility when compared to the progressive motility of spermatozoa of other groups. Significantly lower percentage $(p<0.05)$ motility of spermatozoa was observed among $\mathrm{M} \beta \mathrm{CD}$ treated group when compared to other three groups after freezing and thawing $(\mathrm{p}<0.05)$.

Functional membrane integrity of the spermatozoa treated with $0 \mathrm{mg}$ and $2 \mathrm{mg}$ CLC/120 million spermatozoa or $1 \mathrm{mg}$ and $2 \mathrm{mg} \mathrm{CLC} / 120$ million spermatozoa before cryopreservation did not have significant difference during post equilibration period. Sperms treated with $1 \mathrm{mg} \mathrm{M} \beta C D / 120$ million spermatozoa prior to cryopreservation had significantly lower $(p<0.05 \%)$ percentage of HOS positive spermatozoa than other three groups during post-equilibration and post-thaw stage (Table-2). Functional membrane integrity of the groups treated with $0 \mathrm{mg}, 1 \mathrm{mg}, 2 \mathrm{mg}$ CLC/120 million spermatozoa did not differ significantly during post-thaw stage (Table-2).

Spermatozoa treated with $1 \mathrm{mg}$ CLC/120 $\times 10^{6}$ spermatozoa prior to cryopreservation maintained a significantly higher $(\mathrm{p}<0.05)$ percentage of progressive motile spermatozoa immediately after post-thawing and at $30 \mathrm{~min}$ of thawing than spermatozoa of other groups. CLC groups maintain a higher percentage of progressive motile spermatozoa up to $2 \mathrm{~h}$ of post-thawing. There was no significant difference between the spermatozoa of groups treated with CLC or without CLC at 3 and $4 \mathrm{~h}$ of incubation (Table-3).

\section{Discussion}

Cryopreservation of spermatozoa is a great benefit to animal farming, agriculture and wildlife conservation strategy and simple alternative to embryo freezing for genetic conservation [12]. However, cryopreservation leads to extracellular and intracellular sperm damage result in lipid to protein reorganization and osmotic changes in the membrane.

Cholesterol is a predominant lipid of the sperm membrane and the cholesterol to phospholipid ratio is an important determinant of the sperm membrane fluidity [13]. Cholesterol modulates the fluidity of the membrane as temperature decreases during cryopreservation [14]. Treatment of spermatozoa with cholesterol has been found to reduce the sensitivity of sperm to cooling damage, membrane leakage and minimizing lateral phase separation of lipids [15]. Cyclodextrin, a cyclic oligosaccharide obtained by enzymatic degradation of starch, is used for incorporation or removal of cholesterol from cell membranes [16]. Treatment of spermatozoa with CLC have been shown to result in a 2-3 times increase in the sperm membrane cholesterol content than the normal spermatozoa and ultimately increasing cholesterol: phospholipid ratio [14].

The percentage of progressive motility of spermatozoa of the control group was significantly lower $(p<0.05)$ than the progressive sperm motility observed among spermatozoa of the CLC treated groups. This is in accordance with the results obtained by Konyali et al., in Murcino-Granadina buck and Yildiz et al., in Carp sperm [8,17], Mocea et al., in ram semen [18], Rebecca et al., and Pamornsakda et al., in stallion spermatozoa $[19,20]$ and Crichton et al., in dromedary camel spermatozoa [21] when treated with CLC before cryopreservation. The better post-thaw recovery of progressive sperm motility in spermatozoa treated $1 \mathrm{mg}$ of CLC/120 million cells than control group without CLC is comparable to the values reported by Konyali et al., [8] in bucks after treatment with $1 \mathrm{mg}$ CLC/120 million sperm and Amidi et al. [22] after treatment with $1.5 \mathrm{mg} \mathrm{CLC} / 120$ million sperm and $2.5 \%$ bovine serum albumin. Better post-thaw progressive motility of buck spermatozoa, treated with CLC, was also observed by Farshad et al., [23]. In addition to it a higher dose of CLC ( $2 \mathrm{mg}$ CLC/120 million spermatozoa) put an inhibitory effect on spermatozoa ability for capacitation and to undergo acrosome reaction $[24,25]$.

Purdy and Graham stated that CLC treated spermatozoa, which started with greater membrane cholesterol content prior to cooling, lost only a small

Table-1: Effect of varying cholesterol levels in extender on pre-freeze and post-thaw progressive motility (per cent, Mean \pm SEM) of Malabari buck spermatozoa $(n=12)$.

\begin{tabular}{lcccc}
\hline Stage of processing & Group I & Group II & Group III & Group IV \\
\hline Pre-freeze & $67.67 \pm 1.62^{\mathrm{b}}$ & $75.17 \pm 0.68^{\mathrm{a}}$ & $75.67 \pm 0.94^{\mathrm{a}}$ & $53.5 \pm 3.78^{\mathrm{c}}$ \\
Post-thaw & $40.50 \pm 1.65^{\mathrm{b}}$ & $50.42 \pm 1.17^{\mathrm{a}}$ & $44.58 \pm 2.11^{\mathrm{b}}$ & $16.42 \pm 1.94^{\mathrm{c}}$ \\
\hline
\end{tabular}

Values having different superscript differ significantly $(p<0.05)$. Group I: Control; Group II: Supplemented

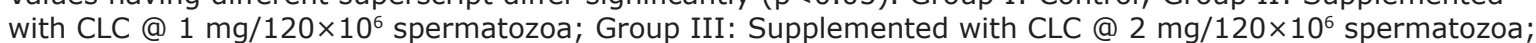
Group IV: Supplemented with Methyl- $\beta$-cyclodextrin @ $1 \mathrm{mg} / 120 \times 10^{6}$ spermatozoa

Table-2: Effect of varying cholesterol levels in extender on pre-freeze and post-thaw HOS response (per cent, mean \pm SEM) of Malabari buck spermatozoa $(n=12)$.

\begin{tabular}{lcccc}
\hline Stage of processing & Group I & Group II & Group III & Group IV \\
\hline Pre-freeze & $58.69 \pm 1.97^{\mathrm{b}}$ & $65.58 \pm 1.68^{\mathrm{a}}$ & $63.69 \pm 0.97^{\mathrm{a}, \mathrm{b}}$ & $46.63 \pm 2.48^{\mathrm{c}}$ \\
Post-thaw & $43.61 \pm 0.92^{\mathrm{a}}$ & $50.05 \pm 1.15^{\mathrm{a}}$ & $49.14 \pm 1.19^{\mathrm{a}}$ & $23.09 \pm 3.20^{\mathrm{b}}$ \\
\hline
\end{tabular}

Values having different superscript differ significantly $(p<0.05)$. Group I: Control; Group II: Supplemented

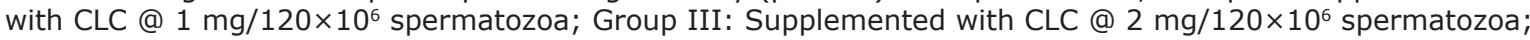
Group IV: Supplemented with Methyl- $\beta$-cyclodextrin @ $1 \mathrm{mg} / 120 \times 10^{6}$ spermatozoa 
Table-3: Effect of post-thaw incubation at $37^{\circ} \mathrm{C}$ on progressive motility (per cent, mean $\pm \mathrm{SEM}$ ) of Malabari buck spermatozoa frozen in extenders with varying level of cholesterol $(n=8)$.

\begin{tabular}{lcccc}
\hline Stage of incubation & Group I & Group II & Group III & Group IV \\
\hline $0 \mathrm{~h}$ & $38.38 \pm 1.97^{\mathrm{c}}$ & $52.5 \pm 2.11^{\mathrm{a}}$ & $46.00 \pm 1.16^{\mathrm{b}}$ & $13.69 \pm 1.39^{\mathrm{d}}$ \\
$30 \mathrm{~min}$ & $23.13 \pm 0.91^{\mathrm{c}}$ & $41.00 \pm 1.48^{\mathrm{a}}$ & $35.63 \pm 2.86^{\mathrm{b}}$ & $5.00 \pm 0.82^{\mathrm{d}}$ \\
$1 \mathrm{~h}$ & $17.50 \pm 2.11^{\mathrm{b}}$ & $28.88 \pm 1.48^{\mathrm{a}}$ & $28.75 \pm 2.95^{\mathrm{a}}$ & $3.38 \pm 0.82^{\mathrm{c}}$ \\
$2 \mathrm{~h}$ & $13.00 \pm 1.91^{\mathrm{b}}$ & $23.38 \pm 2.98^{\mathrm{a}}$ & $22.88 \pm 3.87^{\mathrm{a}}$ & $1.38 \pm 0.73^{\mathrm{c}}$ \\
$3 \mathrm{~h}$ & $8.63 \pm 2.12^{\mathrm{b}}$ & $13.75 \pm 2.61^{\mathrm{a}, \mathrm{b}}$ & $17.13 \pm 4.15^{\mathrm{a}}$ & $0.38 \pm 0.37^{\mathrm{b}}$ \\
$4 \mathrm{~h}$ & $4.5 \pm 1.36^{\mathrm{a}, \mathrm{b}}$ & $10.75 \pm 2.65^{\mathrm{a}}$ & $11.13 \pm 3.18^{\mathrm{a}}$ & $0.00 \pm 0.00^{\mathrm{b}}$ \\
\hline
\end{tabular}

Values having different superscript differ significantly $(p<0.05)$. Group I: Control; Group II: Supplemented

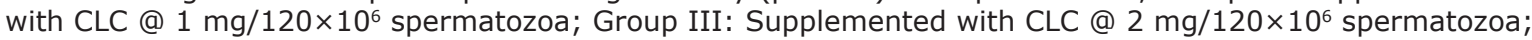
Group IV: Supplemented with Methyl- $\beta$-cyclodextrin @ 1 mg/120×106 spermatozoa

amount of cholesterol during cryopreservation and had a greater amount of cholesterol after thawing than control sperm, which prevented premature capacitation and increased the longevity of cryopreserved sperms [14].

In the present study, treatment of spermatozoa with CLC was found to bring about an increased HOS response (Table-2) than the control group during pre-freeze stage. When sperms are diluted in a hypotonic solution, water moves into the cell fast causing increase in the cell volume which, may cause membrane damage and even cell lysis [26]. No significant difference was observed in the HOS response of spermatozoa in Group I, II, and III after freezing. Incorporation of cholesterol in the sperm membrane improves the sperm cryosurvival by increasing cholesterol: phospholipid ratio in the membrane which reduces lipid aggregation in specific domains within the membrane during phase transition from liquid to gel state and reduces the leakage of cellular components (such as potassium) from the cell [5]. Murphy et al., 2014 and Ahmad et al., 2013 demonstrated increased membrane fluidity obtained after adding CLC to stallion and ram respectively spermatozoa before cryopreservation [27,28].

Membrane damage occurs, when cell membrane undergoes a transition from liquid crystalline to gel [7]. Cold shock resulted in plasma membrane undergoing lipid phase transitions during the cooling process and was inversely correlated with the proportion of cholesterol within the plasma membrane [5]. Hartwig et al., stated that when plasma membrane is exposed to low temperatures, the addition of sufficient levels of liposome containing cholesterol to sperm plasma membrane increases its cryoresistance and as a result it does not undergo a transition phase [29]. Thus, in the present study spermatozoa treated with CLC had better membrane cholesterol content and hence, could withstand cold shock better. Supplementation or removal of cholesterol in the extender for freezing did not manifest in any sperm abnormalities at pre-freeze stage.

Spermatozoa treated with $\mathrm{M} \beta \mathrm{CD}$ showed significantly lower $(\mathrm{p}<0.05)$ percentage of progressive motile and intact plasma membrane than the other three groups during pre-freeze and post-thaw stage.
Removal of cholesterol by M $\beta C D$ has been shown to induce spermatozoa capacitation via tyrosine phosphorylation pathway, thereby shortening the sperm longevity [30]. Similar results were reported by Movassaghi et al., in mouse spermatozoa [31]. The decreased recovery of post-thaw progressive motility in spermatozoa treated with $\mathrm{M} \beta C D$ is likely due to the removal of cholesterol from sperm membrane by cyclodextrin [32]. In addition, treatment with $\mathrm{M} \beta \mathrm{CD}$ has been reported to induce sperm capacitation [30], prior to cooling, making the sperm more sensitive to damage.

CLC groups maintain a higher percentage of progressive motile spermatozoa up to $2 \mathrm{~h}$ of post-thawing. There was no significant difference between the spermatozoa of groups treated with CLC or without CLC at 3 and $4 \mathrm{~h}$ of incubation (Table-3). The better post-thaw recovery of progressive sperm motility in spermatozoa treated $1 \mathrm{mg}$ of CLC/ $120 \times 10^{6}$ cells than control group without CLC is comparable to the values reported by Konyali [8] in bucks after treatment with $1 \mathrm{mg} \mathrm{CLC} / 120 \times 10^{6}$ sperm and Amidi et al., after treatment with $1.5 \mathrm{mg} \mathrm{CLC} / 120 \times 10^{6}$ sperm and $2.5 \%$ bovine serum albumin [22]. Better post-thaw progressive motility of buck spermatozoa, treated with CLC, was also observed by Farshad et al. [23]. Though cholesterol treatment was found to give better post-thaw motility during all stages of incubation when compared to the M $\beta C D$ treated group; it was found that after $2 \mathrm{~h}$ of incubation, the spermatozoa treated with $1 \mathrm{mg}$ of CLC/120 $\times 10^{6}$ spermatozoa had comparable motility with the control group, while the $2 \mathrm{mg}$ CLC $/ 120 \times 10^{6}$ spermatozoa had better motility. By $4 \mathrm{~h}$ of incubation, even this motility was comparable to the control group motility. No references could be found supporting or explaining this observation. The possibility of metabolic products interfering with motility can be ruled out as the buffering capacity of the extended semen is expected to counter the products of metabolism. A possible explanation might be that spermatozoa subjected to cryopreservation have differing ability to withstand cryopreservation stress. In the control group, only the sturdy spermatozoa were able to survive the stress, while in the CLC treated group even the less sturdy spermatozoa survived the cryopreservation stress. However, these 
less sturdy spermatozoa would be subsequently losing their motility when incubated thus leading to a comparable motility after few hours of incubation.

\section{Conclusion}

In conclusion, the percentage of progressive motile sperms was higher $(\mathrm{p}<0.05)$ in spermatozoa treated with $1 \mathrm{mg}$ CLC/120 million sperms after cryopreservation. The spermatozoa treated with $1 \mathrm{mg}$ CLC/120 million sperms was the optimal concentration for goat semen cryopreservation. Addition of $\mathrm{M} \beta \mathrm{CD}$ might remove cholesterol from the sperm membrane making it more prone to cryodamage Future investigations may be directed at assessing the in vivo fertility of CLC treated spermatozoa in sexually healthy Malabari bucks.

\section{Authors' Contributions}

HMH and KNAG planned the study. BS carried out the work. HMH and KLB helped in carrying out the research. All authors participated in draft and revision of the manuscript. All authors read and approved the final manuscript.

\section{Acknowledgments}

The authors are highly thankful to the Dean, College of Veterinary and Animal Sciences, Mannuthy for providing necessary financial assistance and necessary facilities to carry out the research.

\section{Competing Interests} interests.

The authors declare that they have no competing

\section{References}

1. Purdy, P.H. (2006) A review on goat sperm cryopreservation. Small Rumin. Res., 63: 215-225.

2. Moore, A.I., Squires, E.L. and Graham, J.K. (2005) Adding cholesterol to the stallion sperm plasma membrane improves cryosurvival. Cryobiology, 51: 241-249.

3. Chakrabarty, J., Banerjee, D., Pal, D., De, J., Ghosh, A. and Majumder, G.C. (2007) Shedding off specific lipid constituents from sperm cell membrane during cryopreservation. Cryobiology, 54(1): 27-35.

4. Muller, K., Muller, P., Pincemy, G., Kurz, A. and Labbe, C. (2008) Characterization of sperm plasma membrane properties after cholesterol modification: Consequences for cryopreservation of rainbow trout spermatozoa. Biol. Reprod., 78: 390-399

5. Drobnis, E.Z., Crowe, L.M., Berger, T., Anchordoguy, T.J., Overstreet, J.W. and Crowe, J.H. (1993) Cold shock damage is due to lipid phase transitions in sperm membrane: A demonstration using sperm as a model. J. Exp. Zool., 265: 432-437.

6. Bailey, J.L., Lessard, C., Jacques, J., Breque, C., Dobrinski, I., Zeng, W. and Galantino-Homer, H.L. (2008) Cryopreservation of boar semen and its future importance to the industry. Theriogenology, 70: 1251-1259.

7. Darin-Bennett, A. and White, I.G. (1977) Influence of the cholesterol content of mammalian spermatozoa on susceptibility to cold-shock. Cryobiology, 14: 466-470.

8. Konyali, C., Thomas, C., Blanch, E., Gomez, E.A., Graham, J.K. and Moce, E. (2013) Optimising condition for treating goat semen with clolesterol-loaded-cyclodextrin prior to freezing improves cryosurvival. Cryobiology, 67: 124-131.
9. Crockett, E.L. (1998) Cholesterol function in plasma membranes from ectotherms: Membrane specific role in adaptation to temperature. Am. Zool., 38: 291-304.

10. Purdy, P.H. and Graham, J.K. (2004a) Effect of cholesterol-loaded cyclodextrin on the cryosurvival of bull sperm. Cryobiology, 48: 36-45.

11. Jeyendran, R.S., Van Der Ven, H.H., Perez-Pelaez, M., Crabo, B.G. and Zaneveld, L.J.D. (1984) Development of an assay to assess the functional integrity of the human sperm membrane and its relationship to other semen characteristics. J. Reprod. Fertil., 70: 219-228.

12. Zeng, W.X. and Tereda, T. (2001) Effect of methyl- $\beta$-cyclodextrin on cryosurvival of boar spermatozoa. J. Androl., 22: 111-118

13. Yeagle, P.L. (1985) Cholesterol and the cell membranes. Biochim. Biophys. Acta, 822: 267-287.

14. Purdy, P.H. and Graham, J.K. (2004b) Effect of adding cholesterol to bull sperm membranes on sperm capacitation, the acrosome reaction, and fertility. Biol. Reprod., 71: 522-527.

15. Watson, P.F. (1981) Effect of cold shock on sperm cell membranes. In: Morris, G.J., Clark, A., editors. Effect of Low Temperature on Biological Membranes. Academic Press, London, p189-218.

16. Moce, E., Blanch, E., Tomas, C. and Graham, J.K. (2010a) Use of cholesterol in sperm cryopreservation: present moment and perspectives to future. Reprod. Domest. Anim., 45 Suppl 2: 5766.

17. Yildiz, C., Yavas, I., Bozkurt, Y. and Aksoy, M. (2015) Effect of cholesterol-loaded cyclodextrin on cryosurvival and fertility of cryopreserved carp (Cyprinus carpio) sperm. Cryobiology, 70(2): 190-194.

18. Mocea, E., Purdy, P.H. and Graham, J.K. (2010b) Treating ram sperm with cholesterol-loaded cyclodextrins improves cryosurvival. Anim. Reprod. Sci., 118: 236-246.

19. Rebecca, J.M., Lawrence, E.E. and Curtis, R.Y. (2013) The effect of 2-hydroxypropyl-b-cyclodextrin on post-thaw parameters of cryopreserved jack and stallion semen. $J$. Equine Vet. Sci., 33: 272-278.

20. Pamornsakda, T., Pojprasath, T., Suwimonteerabutr, J. and Tharasanit, T. (2011) Effects of cholesterol-loaded cyclodextrins on the quality of frozen-thawed equine epididymal sperm. Cryobiology, 63(2): 90-95.

21. Crichton, E.G., Budhan, S., Billah, M. and Skidmore, A.J. (2015) Cholesterol addition aids the cryopreservation of dromedary camel spermatozoa. Theriogenology, 83: 168-174.

22. Amidi, F., Farshad, A. and Khor, A.K. (2010) Effects of cholesterol-loaded cyclodextrinduring freezing step of cryopreservation with TCGY extender containing bovine serum albumin on quality of goat spermatozoa. Cryobiology, 61: 94-99.

23. Farshad, A., Amidi, F., Khor, A.K. and Rasidi, A. (2010) Effects of cholesterol-loaded cyclodextrin in presence and absence of egg yolk during freezing step on quality of Markhoz buck's spermatozoa. Asian-Aust. J. Anim. Sci., 24(2): 181-189.

24. Aksoy, M., Akman, O., Lehimcioglu, C.K. and Erdem. H. (2010) Cholesterol-loaded cyclodextrin enhances osmotic tolerance and inhibits the acrosome reaction in rabbit spermatozoa. Anim. Reprod. Sci., 120: 166-172.

25. Serin, I., Aksoy, M. and Ceylan, A. (2011) Cholesterolloaded-cyclodextrin inhibits premature acrosomal reaction in liquid stored rabbit spermatozoa. Anim. Reprod. Sci., 123: 106-111.

26. Guthrie, H.D., Liu, J. and Crister, J.K. (2002) Osmotic tolerance limits and effects of cryoprotectants on motility of bovine sperm. Biol. Reprod., 12: 545-551.

27. Murphy, C., English, A.M., Holden, S.A. and Fair, S. (2014) Cholesterol loaded cyclodextrin improves post thaw quality of stallion sperm. Anim. Reprod. Sci., 145: 123-129.

28. Ahmad, E., Aksoy, M., Serin, I., Kucuk, N., Cyalen, A. and Ucan, U. (2013) Cholesterol-loaded-cyclodextrin 
pretreatment of ram spermatozoa protects structural integrity of plasma membrane during osmotic challenge and reduces their ability to undergo acrosome reaction in vitro. Small Rumin. Res., 115: 77-81.

29. Hartwig, F.P., Lisboa, F.P., Hartwig, F.P., Monteiro, G.A., Maziero, R.R.D., C.P. Freitas-Dell Aqua, C.P., Alvarenga, M.A., Papa, F.O. and Dell Aqua, J.A. (2014) Use of cholesterol-loaded cyclodextrin: An alternative for bad cooler stallions. Theriogenology, 81: 340-346.

30. Osheroff, J.E., Visconti, P.E., Travis, A.J., Alvarez, J. and Kopf, G.S. (1999) Regulation of human sperm capacitation by a cholesterol efflux-stimulated signal transduction pathway leading to preotein kinase A-mediated up regulation of protein tyrosine phosphorylation. Mol. Hum. Reprod., 5: 1017-1026.

31. Movassaghi, S., Saki, G., Javadnia, F., Panahi, M., Mahmoudi, M. and Rhim, F. (2009) Effect of methyl- $\beta$-cyclodextrin and cholesterol on cryosurvival of spermatozoa from C57BL/6 mouse. Pak. J. Biol. Sci., 12(1): 19-25.

32. Iborra, A., Companyo, M., Martinez, P. and Morros, A. (2000) Cholesterol efflux promote acrosome reaction in goat spermatozoa. Biol. Reprod., 62: 378-383.

$* * * * * * * *$ 